\title{
DO ALL SPIRAL GALAXIES HAVE A MASSIVE DARK HALO?
}

\author{
M. HONMA ${ }^{1}$, Y. SOFUE ${ }^{1}$ \\ ${ }^{1}$ Inst. Astronomy, Univ. Tokyo, Mitaka, Tokyo 181, Japan
}

We have investigated the masses and extents of dark halos of spiral galaxies by two methods. First, we have reanalyzed HI outer rotation curves so far obtained considering the velocity uncertainties. We confirmed that many HI rotation curves indicate the existence of dark matter to some extent. However, we also found that only few rotation curves provide direct evidences for halos extended beyond 10 disk scale length. On the other hand, recent $\mathrm{HI}$ observations revealed that several galaxies have declining rotation curves that are approximated by Keplerian in the outermost regions. Considering the velocity uncertainties in the outer rotation curves, we found that these declining rotation curves are not rare compared to flat rotation curves. If these declining rotation curves indeed trace the mass truncation, these results indicate that some dark halos have moderate masses that are comparable or slightly larger than disk masses.

Second, we have estimated the total mass-to-light ratio of galaxies based on a newly selected sample of binary galaxies. The average mass-to-light ratio is consistent with the dark halos with moderate masses and sizes.

\section{References}

Honma M., Sofue Y. 1997, PASJ 49, 539 